\title{
Estudio y embarazo en la universidad. Un reto difícil de superar*
}

\author{
Study and Pregnancy During College. \\ A Stressful Challenge to Overcome
}

\author{
Andrea Hernández Quirama** \\ ORCID: 0000-0003-1955-0566 \\ Flor de María Cáceres Manrique \\ ORCID: 0000-0002-4796-6190 \\ Carolina Rivero-Rubio \\ ORCID: 0000-0002-5508-4286 \\ Universidad Industrial de Santander, Colombia \\ Recibido: 13 de noviembre de 2019 \\ Revisado: 17 de febrero de 2020 \\ Aceptado: 22 de abril de 2020
}

\section{Resumen}

La vivencia del embarazo depende de la etapa del ciclo vital, de su planificación, del apoyo familiar y los recursos económicos con los que se cuenta. En edades tempranas condiciona el nivel educativo, económico y social de las madres, los padres y los hijos. Se realizó un estudio cualitativo, con abordaje interpretativo y con métodos de la teoría fundamentada. Se aplicaron 27 entrevistas a 19 madres y 8 padres estudiantes universitarios. El muestreo fue mediante la técnica de bola de nieve. Los resultados indican que solo un embarazo fue planeado. Para hombres y mujeres fue difícil asimilar la noticia, pero la aceptan con el avance del embarazo. Se presentan repercusiones en el rendimiento académico, alteraciones en estado de salud y preocupación económica. En conclusión, el embarazo en estudiantes universitarios genera alteraciones en su proyecto de vida y trae consigo la adopción de nuevos y múltiples roles que complejizan la finalización exitosa de sus estudios superiores, enfrentándolos a procesos de retención o deserción escolar.

Palabras claves: Embarazo, Estudiantes, Universidad, Experiencia

Artículo de investigación. Citar como: Hernández, Q. A., Cáceres, M. F. y Rivero-Rubio, C. (2020). Estudio y embarazo en la universidad. Un reto difícil de superar. Diversitas: Perspectivas en Psicología, 16(2), 353-363. https://doi.org/10.15332/22563067.6318

Autor de correspondencia: Andrea Hernández Quirama, Profesora, Universidad Industrial de Santander. Dirección postal: Calle 9 Cra 27. Edificio de Administración 2 - Oficina 512, Bucaramanga, Colombia. Correo electrónico: ahernanq@uis.edu.co 


\section{Abstract}

The experience of pregnancy depends on the stage of the life cycle, its planning, family support, and economic resources. At early ages, pregnancy conditions aspects such as the social, economic, and educational levels of mothers, fathers, and children. A qualitative study was carried out, with an interpretative approach, and framed in the grounded theory. We conducted 27 interviews to 19 mothers and 8 fathers who were university students. The sampling was done through the snowball technique. Results showed that only one pregnancy was planned. For men and women, it was difficult to assimilate the news. However, they accepted it with the progress of the pregnancy. There were repercussions on the academic performance, alterations in health, and economic concerns. In conclusion, pregnancy in college students produces alterations in their life project and brings with it the adoption of new and multiple roles that make more complex the successful completion of their higher education, exposing them to processes of retention or dropout.

Keywords: pregnancy, students, university, experience.

\section{Introducción}

El embarazo es una etapa del ciclo vital que genera cambios en diversos ámbitos en la vida de las personas. Por una parte, la noticia es asumida de manera diferente dependiendo si se había planificado o no; para quienes no lo esperaban significa un cambio abrupto en sus vidas, y esto se complejiza de acuerdo a la etapa en la que se encuentran. En este caso, para las mujeres y hombres jóvenes (estudiantes universitarios), por el momento vital que cursan el embarazo implica ajustes dramáticos en sus vidas, tanto en lo académico, en lo económico, en su salud, en sus familias y especialmente, en asumir un nuevo rol: el de ser padre o madre, rol que no se tenía previsto.

Es entonces como el inesperado estado de embarazo y sus abruptos cambios, pueden incidir en el abandono de los estudios universitarios. Esto, para dedicarse a trabajar y así conseguir recursos económicos para la manutención de los hijos, además de la demanda de tiempo para el cuidado y crianza. Este tipo de decisiones afectan de manera considerable a las madres y los padres estudiantes, pues al desertar del sistema educativo, se pospone o en ocasiones se cancela la opción de ser profesional, y por tanto, se minimizan las oportunidades de conseguir un trabajo con garantías laborales que le signifiquen estabilidad reflejando esto en su calidad de vida personal y familiar. Lo que coincide con los estudios realizados por Rodríguez Urrego (2019) y Núñez, Chavarría y Molina (2011).

El fenómeno de la maternidad y la paternidad en la adolescencia y juventud ha sido estudiado principalmente a partir de la Conferencia Internacional sobre Población y Desarrollo, El Cairo 1994, donde se estimó que el embarazo a temprana edad condicionaba el nivel educativo, económico y social al limitar en alto grado las oportunidades de educación y empleo, lo cual producía efectos negativos sobre la calidad de vida de los padres y sus hijos (Naciones Unidas, 1994).

Adicionalmente, la noticia del embarazo puede generar respuestas de rechazo por parte de las familias de origen, quienes usualmente ante el impacto de la noticia manifiestan a las y los jóvenes que no se continuará con el apoyo económico. Esto, dado que por la gestación se les asigna la condición de adultos a los nuevos padres, además de un sinnúmero de responsabilidades que, estén o no preparados, deben asumir. Estas condiciones generan un aumento en la preocupación de las nuevas madres y padres. Se ha encontrado que esta situación puede 
llegar a modificarse, en especial con el nacimiento del hijo, pues los abuelos se "enternecen" y le brindan cuidados al bebe, como se evidencia por el estudio realizado por Huerta (2019). Para las madres y padres estudiantes permanece la recarga inesperada de responsabilidades que inciden en todos los ámbitos de sus vidas. Por lo anterior, se realizó un estudio que evidencia las situaciones vividas por las madres y los padres estudiantes de la Universidad Industrial de Santander con el embarazo.

\section{Metodología}

Se realizó un estudio cualitativo, desde el paradigma interpretativo, cuyo objetivo fue explorar las vivencias asociadas al embarazo de estudiantes universitarios que son madres y padres. Se utilizó como método de análisis la teoría fundamentada según lo expuesto por Strauss y Corbin (1998).

Este estudio tuvo las siguientes fases: 1) preparatoria, 2) de trabajo de campo y 3) analítica (Rodríguez, Gil y García, 1996). La fase preparatoria tuvo dos momentos: uno reflexivo, donde se revisó la literatura existente relacionada con el embarazo y en el segundo, de diseño, se elaboraron las guías de entrevista. Se caracterizó la población estudiantil acorde a los siguientes criterios: estudiantes de pregrado de la Universidad Industrial de Santander (Colombia) activos, madres, padres o gestantes, menores de 24 años.

En la fase de trabajo de campo se identificaron, contactaron y citaron a entrevista los participantes vía telefónica. Durante el encuentro se expuso el objetivo del estudio y sus alcances. Una vez aceptada la participación se procedió a leer el consentimiento informado verbal, aprobado por el Comité de ética de la Universidad. El muestreo se realizó con la técnica de Bola de Nieve hasta que se logró la saturación de la información, la cual se presentó cuando la información se empezó a repetir. Se aplicaron en total 27 entrevistas a 19 madres y 8 padres, que no eran parejas entre sí; sus edades oscilaban entre los 18 y 24 años, de diferentes programas académicos y diferentes semestres.

Paralelo a la recolección de la información, donde se utilizó la técnica de entrevista semiestructurada, se llevó a cabo el análisis de la misma, el procesamiento de la información se realizó con $N V i v o{ }^{\circledR}$ versión 11. La teorización se hizo a medida que se realizaban las entrevistas, que se transcribieron, codificaron y categorizaron, lo que permitió integrar y refinar las categorías, organizadas en un esquema categorial alrededor de un concepto explicativo central. Se buscó un muestreo teórico que a partir de los datos permitiera desarrollar teoría sustantiva y determinar el nivel de saturación de las categorías. Para lo cual se siguió la metodología planteada por Strauss y Corbin (1998) que comprende, descripción, ordenamiento conceptual y teorización.

\section{Resultados}

\section{Estudiantes Madres: No esperaba quedar embarazada}

La maternidad en la juventud como categoría analítica muestra su complejidad por las implicaciones que ocasiona a nivel individual, familiar y social. Tal es su importancia que en Colombia, la Política Nacional de Sexualidad, Derechos Sexuales y Reproductivos, que toma como sustento la Encuesta Nacional de Demografía y Salud (ENDS) 2010, plantea atender las necesidades educativas y de servicios de los adolescentes con el fin de que puedan asumir de modo positivo y responsable la sexualidad y reproducción (Ministerio de Salud y Protección Social, 2014.

Son diversos los factores protectores y de riesgo en el embarazo adolescente, según la Mesa Técnica de Estudios a Profundidad de Profamilia, como factores protectores se encuentran la asistencia escolar, el desarrollarse en un hogar nuclear, el clima educativo del hogar, las condiciones socioeconómicas y la educación sexual (Profamilia, 2013). Así, es característico de los estudios relacionar el embarazo a temprana edad con el aumento de las desigualdades sociales y la pobreza, como los planteados por (García Hernández, 2014; Mazuera y Albornoz, 2017). No obstante, más allá de estas manifestaciones, existe gran interés por comprender los significados de la maternidad, entendida como un factor de cambio subjetivo que tiende a generar crisis por 
las transformaciones que se producen tras los nuevos eventos y roles que implica esta transición en la vida de la mujer (Velásquez, 2009). Esta condición se agudiza cuando se encuentran dos etapas en el ciclo vital de las mujeres jóvenes: la maternidad y el estudio.

En nuestra muestra, la totalidad de las estudiantes entrevistadas - a excepción de una participante que manifestó haber planeado el embarazo- coinciden en las repercusiones que se presentaron en su rendimiento académico, mostrando un descenso notable, y la mayoría tuvieron que aplazar semestres y ausentarse de la vida académica por un tiempo.

Adicional a la vida académica, las estudiantes presentaron cambios en su estado de salud física y mental, evidenciados en las transformaciones en su cuerpo, el proceso de aceptación del embarazo, la presión personal, de pareja, familiar y social, que según lo que ellas manifestaron en las entrevistas, generó en ellas tristeza y síntomas depresivos, es así como aparece un cúmulo de emociones que deben asumir y enfrentar.

Las mujeres se enfrentaron a la situación de dar la noticia a sus familiares, quienes usualmente manifiestan frustración ante la afectación en el proyecto de vida de la estudiante, ahora madre. Estas reacciones y situaciones se repitieron con diferentes matices y respuestas por parte de la pareja y de los amigos.

Fue común encontrar que las mujeres no esperaban atravesar por esta situación, particularmente en este periodo del ciclo vital. Sin embargo, a pesar de los sentimientos iniciales de perplejidad, tristeza, miedo y ansiedad que pudo generar la noticia por la incertidumbre frente al futuro, la crianza y la subsistencia; el apoyo de la pareja y la familia fue determinante para afrontar esta nueva etapa, la cual, para la mayoría de las estudiantes comenzó con sentimientos encontrados y temores.

La verdad fue sorpresa, no me lo esperaba y pues yo era una de las que decía que yo no quería tener hijos. La verdad me daba miedo, me daba miedo porque yo dije, bueno ¿ahora qué hago?, pensé en irme de la casa. Fueron muchos sentimientos encontrados. Nosotros llevábamos muy poco tiempo y él fue el primero al que yo le conté y pues bien. Él contento. Yo era la asustada. Pero sí, él se puso contento apenas supo, porque él no tenía hijos entonces quería tener hijos. Estudiante mujer Facultad Ingenierías Fisicoquímicas.

En otro de los relatos se evidencia como ante la sospecha de embarazo, se espera con ansia que la noticia se modifique. Sin embargo, ante la ratificación de la misma se asume con resignación y expectativa de poder asumir el cambio.

Me dijo, esperemos que pasen los dos días y pasaron dos días después de que no me llegaba el periodo y me dijo, amor, usted está embarazada y yo: ¿qué? Dijo, sí, vamos a hacernos la prueba y yo dije, no, después de que cumpla años. No, él estaba más contento y yo estaba más triste. ¿Ahora yo cómo iba a hacer para seguir estudiando?, ¿quién me iba a cuidar la niña?, bueno, en fin. Pero él decía que no, que sí, que eso iba a poder. Estudiante mujer Facultad Ingenierías Fisicoquímicas.

Ante la noticia del embarazo la primera reacción es de sorpresa, de negación, de tristeza y angustia ante lo que implica la maternidad mientras se está estudiando, al pasar el tiempo de gestación poco a poco se empieza a aceptar la noticia y cuando nace el hijo, la reacción inicial se modifica por emoción y alegría ante su presencia.

Cuando me enteré ninguno lo esperaba conscientemente por decirlo así, entonces yo sí cuando lo recuerdo eso me da un poco de tristeza, el primer impacto no fue felicidad, ahora sentirme feliz y recordar que en ese momento es un poco extraño. Muchos miedos al principio, uno no tiene ni idea de lo que va a venir después, aunque estés en el último mes no vas a tener idea cómo va a ser cuando nazca tu bebé. Lo otro fue comentarles a nuestros papás, fue otra fase, pero la superamos con facilidad y con rapidez. Contamos con suerte de tener su respaldo. Estudiante mujer Facultad de Ciencias.

También se encontraron respuestas de aceptación, apoyo y felicidad por parte de la pareja de jóvenes 
estudiantes y de sus familias, lo que significa un alivio para la madre, que experimenta el respaldo de las personas cercanas a su vida.

En cuanto al sentimiento pues fue muy feliz, o sea yo afortunadamente conté con el apoyo de mi esposo que en ese momento era mi novio. Con el apoyo de la familia de él, con el apoyo de mi familia, sobre todo, entonces pues en realidad no tuve un embarazo triste. Estudiante mujer Facultad de Ciencias Humanas.

La noticia en ocasiones se asigna al destino, a algo que se debía vivir, situación que se respaldó con la respuesta de la familia asociada a las creencias religiosas, esto facilitó la aceptación, que puede entenderse como resignación del embarazo.

Fue algo inesperado, quizá no estaba preparada en ese momento. Pues igual todo el embarazo estuve como preocupada, como ¿qué iba a hacer con mi futuro? Gracias a Dios siempre estaban mis padres, que ellos me daban la moral y eso. Entonces esto, en ese aspecto no estuve tan sola, estuve con ellos y pues al principio, realmente, no quería, no era algo esperado y quizá de pronto en ese momento el bebé sintió ese rechazo. Ya después me fui haciendo a la idea que Dios así lo quiso y tenía que recibirlo. Estudiante mujer Facultad Ingenierías Fisicomecánicas.

Entre los hallazgos se destacó que, aunque algunas mujeres continuaron con sus parejas y fortalecieron su relación, la tendencia fue que se generaron rupturas. En algunos casos las mujeres manifestaron cómo esto las llevó a sentirse solas, a pesar de contar con el apoyo de su familia de origen.

No tuve un embarazo muy alegre, no tengo relación con el papá de la niña, nunca la tuve, entonces fue como vivirlo sola, pues al lado de mis papás obviamente, pero a veces hace falta como esa pareja, pues lo tuve como muy triste, siempre estuve muy triste en el embarazo, lloraba mucho, debe ser por las hormonas o algo así, pero era complicadísimo, tuve un embarazo complicado. Estudiante mujer Facultad de Ciencias Humanas.
Por lo general, la reacción con la familia ante la noticia fue en un primer momento de frustración, pues el embarazo en este periodo vital tiende a asumirse en como un fracaso del proyecto ideado por la familia para la joven.

Por mi familia sí fue algo terrible, embarazada a los 18 y obviamente que el estudio, que no puede seguir estudiando. Bueno eso es lo que piensan todos. Para mis papas fue algo impresionante porque como siempre le dicen: usted debe terminar de estudiar, si usted tiene un hijo no puede seguir estudiando, hasta ahí le llegó su vida. Al principio ellos asumieron una posición de rechazo conmigo, pero después como a la semana, perdónenos, fue algo de momento, de la noticia y después ellos fueron un apoyo muy grande. Estudiante mujer Facultad de Ciencias Humanas.

La manifestación de resignación de la familia fue interpretada por las estudiantes como una expresión de apoyo y, por tanto, si el embarazo se estaba ocultando, es a partir de comunicar la noticia que los cambios físicos se percibieron más evidentes.

Cuando ya tenía como cuatro meses y medio yo le dije a mi mamá y mi mamá dijo que bueno, que ya qué podía hacer, que tenía que después terminar la carrera. Apenas le conté se notó la barriga. Estudiante mujer Facultad de Ciencias.

Fue común encontrar a las mujeres sentir mayor confianza con sus madres para contar su situación. Las jóvenes buscaron comprensión y solidaridad en la madre, cuya primera ayuda se centró en transmitir la noticia a los demás miembros de la familia. La figura materna se percibe comprensiva, contraria a la paterna que se asoció a la autoridad y rudeza, con esto se evidenciaron rasgos de una cultura dominante y patriarcal.

Vivo con mi mamá y con mi padrastro, pero entonces fue un poquito complicado porque ella era la única que sabía, pues como mi papá es el que me paga la universidad, él no vive conmigo y él si es más riguroso, entonces él no sabía, él se enteró cuando yo ya tenía siete, ocho meses y a mí nunca se me notó, creo que fue por 
eso, como por esconderle. Entonces yo estaba esperando a que se terminara el semestre por miedo a que mi papá me dijera no le pago más, entonces yo dije voy a terminar el semestre y pues cualquier cosa se puede hacer para el otro semestre. Pero sí fue un poquito complicado, porque no me podía encontrar con alguien conocido porque de una vez va y le dice a mi papá, pero ya cuando mi papá se enteró. Sí lo tomó mal, obviamente, le dio duro, pero ahora me apoya. Estudiante mujer Facultad Ingenierías Fisicomecánicas.

Las estudiantes esperaban que al dar la noticia del embarazo en la familia se presentara resistencia, y ante una respuesta de aceptación quedaron sorprendidas. Las madres de las estudiantes fueron más conciliadoras que los padres, a quienes la noticia de su hija embarazada les afectó de manera sustancial.

Mejor de lo que esperaba, yo esperaba que fuera más terrible. Obviamente las aclaraciones que le hacen a uno, que eso es responsabilidad suya, todo por cuenta de ustedes dos. Mi papá si fue un poquito más difícil por obvias razones de nuestra cultura y con él sí durante el embarazo hubo distanciamiento, porque no vivimos en la misma ciudad, entonces era un poquito distante, las veces que iba a visitarlos cada vez iba acercándose más, ya ahora con el bebé es otro cuento. Estudiante mujer Facultad de Ciencias.

El embarazo tuvo implicaciones en el estado de salud de las estudiantes. Aquellas que no pudieron terminar el semestre académico que se encontraban cursando en el momento de la noticia, se vieron forzadas por su situación a cancelarlo. Esto implicó que en promedio las mujeres se ausentaran entre dos y tres semestres académicos, es decir, entre un año y año y medio para atender su situación de salud durante el embarazo y luego destinar un tiempo exclusivo a la crianza inicial.

Yo supe que estaba embarazada en enero y ahí corté, me dio muy duro cuando supe que estaba embarazada anímicamente y de salud, porque ahí empecé los mareos, vómitos, todo, me dio muy duro el embarazo, preferí no volver a la universidad hasta que no tuviera el bebé.
Esperé y hasta este semestre volví, duré tres semestres por fuera. Estudiante mujer Facultad de Ciencias Humanas.

En ocasiones el embarazo representó para algunas estudiantes alteraciones en su estado de salud, haciendo que debieran asistir al sistema de salud de forma continua, no sólo por los controles prenatales sino también porque se sentían enfermas, y esto las obligó a aplazar sus estudios, lo que a su vez tuvo una repercusión negativa en la culminación de su carrera profesional.

Cancelé el semestre porque aparte de que no iba bien en rendimiento cuando quedé embarazada, pues me quitaba tiempo el hecho de ir a las citas con el médico y decidí cancelar semestre, cancelé uno y paré dos mientras el niño. Estudiante mujer Facultad Ingenierías Fisicomecánicas.

\section{Estudiantes Padres: No sabíamos que íbamos a ser padres tan jóvenes}

El embarazo para los estudiantes hombres, al igual que para las mujeres fue algo inesperado, lo que ocasionó cambios en sus expectativas y necesidades, pasando de la libertad propia de la juventud y de sus estudios a asumir obligaciones y responsabilidades de la etapa adulta. Ellos se vieron conminados a buscar un trabajo para obtener remuneración, así fuera esporádica, que aportara de alguna manera al sostenimiento de su hijo o hija. Estas nuevas ocupaciones de trabajador, padre y además estudiante generaron transformaciones contundentes en sus vidas.

Otra modificación en los jóvenes se dio a través del grado de acompañamiento del estudiante padre a la madre durante la gestación, esta compañía dependió del tipo de relación previamente establecida y pudo reforzar o terminar esta relación. Esto tuvo implicaciones directas en la manera que se asumió su paternidad.

Un aspecto importante para la dinámica de la paternidad fue la comunicación de la noticia del embarazo a la familia de la joven, lo que constituyó una muestra de responsabilidad esperada socialmente 
por parte del hombre y por tanto de aceptación y tranquilidad para la familia de la madre, pues se asumió que no estaría sola con la maternidad.

Aunque al principio, el embarazo generó temores por la expectativa del futuro y los cambios imprevistos, para algunos estudiantes sus creencias religiosas les permitieron comprender y aceptar esta paternidad temprana, y asumieron los hijos como un designio divino.

Mi Dios dice que los hijos no están programados. En cualquier momento de la vida uno puede ser padre, pero si usted tiene planes, hay que tratar de cuadrarlos con la niña. Fue algo drástico porque no sabíamos que íbamos a ser padres tan jóvenes. Estudiante hombre Facultad de Ingenierías Fisicomecánicas.

Luego del temor inicial por el embarazo, experimentaron una etapa de aceptación y resignación, donde los miedos pasaron a convertirse en alegría por parte de algunos estudiantes, esto asociado a sus deseos de ser padres, lo que pudo verse explicado como una prueba de su masculinidad.

Fue algo obviamente inesperado porque ninguno de los dos lo tenía planeado, pero pues lo recibimos y lo asumimos de una forma muy madura que de una forma u otra lo aceptamos. Ya está ahí, entonces tenemos que sacarlo adelante como sea, a medida que pasa el tiempo uno se da cuenta de la magnitud de todo. Estudiante hombre Facultad de Ingenierías Fisicomecánicas.

Fue así como el embarazo inesperado terminó siendo aceptado y luego ante los primeros exámenes médicos surgió la confirmación de la paternidad.

Pues pasó y ella feliz, contenta con el bebé. Le hicieron ya una ecografía y ya se pudo identificar la cabecita, la columna vertebral. Yo la acompañé. Estudiante hombre Facultad de Ingenierías Fisicoquímicas.

La felicidad inicial que experimentaron algunos estudiantes al recibir la noticia, se presentó en medio de los señalamientos a los que se vieron expuestos, los cuales fueron más fuertes cuando el embarazo se dio con menores edades. Estos embarazos tempranos se dieron en algunos casos de forma temerosa, teniendo en cuenta el momento del ciclo vital en el que se presentaron y estuvieron asociados a los comentarios y críticas a los cuales se vieron expuestos, por la corta edad en la que se encontraban y el afán de las familias ante la responsabilidad, de la que al parecer los jóvenes no eran conscientes en su momento.

La verdad cuando yo supe que ella quedó embarazada yo me puse feliz. Irónico, pero me puse feliz, no sabía lo que se venía encima, pensaba que era más fácil. A ella si le pegó duro, pues por diferencia de edades, yo acababa de graduarme y ella estaba cursando bachillerato, llevábamos una relación de tres años cuando quedó embarazada y pues muy preocupada, muy alterada por la situación. Ella tenía 16 años y yo tenía 17 años, los dos menores de edad y claro los futuros de la familia prácticamente recaían sobre nuestros hombros, entonces nos señalaron mucho al principio. Estudiante hombre Facultad de Ingenierías Fisicomecánicas.

La juventud, la inexperiencia, el desconocimiento de lo que implica la paternidad, generó temores que fueron exacerbados por las personas del entorno, quienes indagaron a los jóvenes sobre el futuro, sobre el cómo iban a responder y a organizarse para asumir el nuevo e imprevisto rol en sus vidas. Además, con el embarazo aparecieron las primeras responsabilidades que se asumieron de manera tradicional al rol masculino, surgieron las primeras obligaciones de proveeduría, ligadas a las necesidades propias de la etapa de gestación.

La experiencia del embarazo en los estudiantes trajo preocupaciones desde el momento de comunicar la noticia. Entonces, adicionalmente a que al joven estudiante se le asignó la responsabilidad económica, como parte fundamental de la paternidad, también expresaron que tenían el compromiso de comunicar la noticia del embarazo a la familia de la madre, con lo cual se esperaba una mejor comunicación y aceptación con la familia materna del hijo o hija. Esto al expresar que iban a responder por el hijo que venía en camino, les producía tranquilidad y de una u otra forma mejoraron las relaciones entre los jóvenes y las familias. 
Yo hablé con los papás, les comenté y bien. Como yo he sido responsable me han aceptado. Estudiante hombre Facultad de Ingenierías Fisicoquímicas.

\section{Discusión}

El embarazo en las mujeres jóvenes genera una dinámica que trae consigo implicaciones drásticas en su proyecto de vida, en especial cuando se encuentran cursando estudios universitarios. Se entiende que a mayor edad, la planificación del embarazo y el nivel de estudios de la madre, mejorará su actitud y percepción del embarazo y de la maternidad. El factor que más incide en la adaptación y la actitud es el nivel de estudios de la mujer. Las mujeres de mayor nivel de estudios refieren una mejor percepción de su imagen corporal debido a que presentan una mayor autoestima (Molinero et al., 2007).

Además de lo anterior, el embarazo y la maternidad en mujeres estudiantes universitarias genera tensiones por la multiplicidad de tareas atribuidas a los roles de mujer, estudiante, hija y madre. Los cambios en el proyecto de vida pueden traer como consecuencia la deserción escolar, inestabilidad en las relaciones de pareja, posibilidad de más embarazos, problemas familiares, falta de oportunidades, cambio del rol de estudiante y problemas en el cuerpo (Hernández y Orozco, 2011). De igual forma, en estudiantes universitarias colombianas han sido descritas la deserción estudiantil (23.5\%) y la pérdida parcial de la salud (29.4\%) como consecuencias del embarazo mientras se cursan los estudios superiores (Díaz, González y Ketty, 2010).

Frente a las repercusiones del embarazo y la maternidad en la vida académica de las estudiantes universitarias, Preciado, Acuña y García (2005) afirman que estas repercusiones se relacionan con desajustes fisiológicos como tensión arterial baja, somnolencia, mareos, náuseas y las necesidades de cuidado que demanda el recién nacido; actitudes que en el mundo de la ciencia corresponden al espectro de la experiencia subjetiva que se contrapone con la objetividad y la razón propias del quehacer científico y profesional.
Para Palomar (2009), en el mundo académico se interrelacionan tres dimensiones: la lógica interna de este ámbito, la subjetividad que tiene lugar en este espacio y la maternidad de las académicas. En este contexto, las mujeres parecen responder a exigencias de la tradición y las costumbres propias del orden de género y se encuentran entonces con una experiencia de maternidad compuesta de altibajos entre el trabajo académico y la maternidad.

Es claro que desempeñar el rol materno repercute en el rendimiento académico de las mujeres estudiantes universitarias. En un estudio descriptivo con enfoque cualitativo se encontró que de una muestra de 68 estudiantes embarazadas y madres de primer, segundo y tercer año de la carrera de Enfermería de la Universidad de Cuenca en Ecuador, el 57.4 \% de las encuestadas consideró que cambió su dinámica de estudio con el embarazo y maternidad, el $35.3 \%$ desertó de la carrera, el 25.0 \% tuvo variaciones en su rendimiento académico y el 14.7 \% perdió el año escolar (Cuenca y Espinoza, 2014).

En el ámbito personal, las mujeres logran la aceptación del nuevo estado a medida que aumentan las semanas de gestación (Estupiñán y Rodríguez, 2009). Esto en gran medida está determinado por la experiencia física, biológica, social, espiritual y emocional de la joven, quien con apoyo logra aceptar más fácilmente su estado al reducir la incertidumbre y miedos que se desencadenan tras la noticia. Lo que coincide con los estudios realizados por Morales y colaboradores (2013) y Estupiñan y Vela (2012). En el presente estudio, se resalta que las creencias religiosas influyen en los sentimientos y decisiones hacia el embarazo y la maternidad, los cuales pueden considerarse como un designio divino.

La respuesta de la familia ante la noticia del embarazo y posterior apoyo influye en la experiencia que las estudiantes tienen sobre el embarazo e incide en la continuación o aplazamiento de su proyecto académico, como lo mencionan Estupiñán y Rodríguez, el apoyo social se convierte entonces en un determinante de la felicidad y el éxito en el proyecto educativo materno (2009). En el estudio de Preciado y colaboradores, se evidencia que a pesar de los esfuerzos de la familia y de la mujer 
por continuar el proyecto educativo, se presentan desajustes fisiológicos que llevan a que las universitarias posterguen su proyecto educativo (2005).

Al igual que la mayoría de las mujeres, ninguno de los padres jóvenes refirió el embarazo como planeado o deseado, para todos fue un evento sorpresivo. Es así como los cambios que deben afrontar ante la paternidad ponen a prueba su resistencia y formación como hombres (Duarte, 2016). Esta situación se va modificando en cuanto avanza el embarazo, pues a medida que el tiempo de gestación es mayor, se presenta más conciencia de la presencia del niño (Estupiñán y Rodríguez, 2009).

El embarazo para los jóvenes estudiantes es en la mayoría de los casos una situación no planeada, ante la que experimentan la presión de lo que la sociedad espera de la masculinidad hegemónica, asumir la responsabilidad de tener descendencia y familia (Duarte, 2016). Aunque los hombres sientan temores y tensiones ante una realidad para la cual no estaban preparados, lo que les generó emociones ambivalentes y toma de decisiones que afectaron su proyecto de vida. Situación que coincide con la presentada en las mujeres estudiantes.

Dado que la noticia del embarazo es inesperada, complejiza su vida, los sentimientos que expresan los estudiantes son de angustia, incertidumbre de los cambios que implica el nacimiento del hijo (Briones y González, 2015), lo que ubica a los jóvenes ante la responsabilidad de proveeduría socialmente asignada a los hombres, se constituye así en la entrada al mundo adulto (Toneli, 2006). Donde deben asumir responsabilidades inesperadas para los jóvenes (Antunes, Pereira y Ferreira, 2012). La dimensión económica se convierte en su principal preocupación previendo la crianza y cuidado (Hernández y Rojas, 2018), además, deben cubrir las necesidades del hijo y los gastos asociados al embarazo, como los controles de salud. Conseguir ingresos se convierte en un imperativo para los estudiantes (Briones y González, 2015).

Los imaginarios del rol paterno como proveedor, cuestionan al joven sobre su capacidad para desempeñarlo con éxito pues en estos imaginarios el joven debe hacerse cargo no solo del hijo sino de la madre adolescente sin haber adquirido independencia económica (Botero y Castrillón, 2015). Lo que implica una sobre carga inesperada para el joven estudiante.

Otra tarea clave, y asignada a los estudiantes, es la de comunicar la noticia del embarazo en la familia de su pareja, lo cual denota que asumirá la responsabilidad del hijo. Esto coincide con el estudio de Escobar (2012) en donde la transmisión de la noticia se considera un paso clave para asumir su paternidad.

En cuanto a la relación de pareja, el embarazo influye en la decisión de ruptura o consolidación de la relación, es así como surge la decisión de convivir o alejarse de la pareja (Duarte, 2016). Además, la experiencia del embarazo en los estudiantes se encuentra fuertemente asociada al apoyo del grupo familiar primario, lo que incide en la permanencia en la universidad, subsistencia y posibilidades futuras. Se ponen de cara múltiples roles, a veces contradictorios, como lo son el de jóvenes, estudiantes, padres o madres y trabajadores, los cuales constituyen un reto para los estudiantes universitarios y complejizan la conclusión exitosa de sus estudios.

\section{Conclusiones}

En síntesis, la etapa del embarazo tiene vivencias comunes tanto para las estudiantes madres como para los padres, relacionadas con la no planeación del mismo, los elementos sorpresivos al enterarse de la gestación que involucran sentimientos contradictorios, los temores frente a la respuesta familiar, y la asunción de nuevos roles, entre otros. Para las mujeres, la experiencia del embarazo está determinada por el apoyo que reciban de su familia de origen y su pareja, quienes contribuyen a reducir la incertidumbre ante la crianza, la economía, subsistencia y el futuro. Se percibe redireccionamiento del proyecto de vida que inicia con la suspensión de las actividades académicas. Además se presentan cambios relacionados con el embarazo en la salud física y mental de las estudiantes.

Por su parte, el embarazo para los jóvenes padres se expresa en la dualidad del temor y aceptación. También significa decidir continuar o no una relación de pareja, además del inicio de un proceso de 
asumir responsabilidades asignadas socialmente a la paternidad, algunas de tipo económico, como aportar monetariamente a los cuidados y manutención de la madre embarazada; y otras que evidencian cumplimiento y compromiso con su nuevo rol, como el comunicar la noticia del embarazo a la familia de la madre.

En ambos casos, un elemento crítico consistió en el apoyo recibido por sus núcleos familiares, que determinó la posibilidad de continuar estudiando, armonizó la adaptación ante esta etapa y facilitó el desarrollo de nuevas maternidades y paternidades.

\section{Referencias}

Antunes, E., Pereira, L. y Ferreira, D. (2012). Los significados de la paternidad para los hombres jóvenes en los alrededores de São Paulo-Brasil. Cultura de los cuidados, 16(33), 55-66. https:// doi.org/10.7184/cuid.2012.33.08

Botero, L. y Castrillón, L. (2015). La experiencia de la paternidad en adolescentes. Revista Virtual Universidad Católica del Norte, 46, 89-101.

Briones, V. y González, J. (2015). Estudio exploratorio de paternidad en padres universitarios de la Pontificia Universidad Católica de Valparaíso. Viña del Mar: Pontificia Universidad Católica de Valparaíso.

Cuenca, K. y Espinoza, M. (2014). Repercusiones del embarazo y la maternidad en el rendimiento académico de las estudiantes de la carrera de Enfermería de la Universidad de la Cuenca. Cuenca: Universidad de Cuenca. Recuperado de: http://dspace.ucuenca.edu. ec/handle/123456789/21245

Díaz, S., González, F. y Ketty, R. (2010). Historia de embarazos en estudiantes de programas de salud en una universidad pública del Caribe colombiano. Revista Clínica de Medicina de Familia, 3(3), 163-169.

Duarte, M. (2016). Embarazo y paternidad juvenil en el departamento del Cesar ¿Y dónde está el padre? Revista Colombiana de
Ciencias Sociales, 7(2), 425-447. https://doi. org/10.21501/22161201.1808

Escobar, J. D. (2012). La experiencia de la procreación en adolescentes varones de la ciudad de Medellín. Revista de Psicología. Universidad de Antioquia, 4(1), 75-85.

Estupiñán, M. R. y Rodríguez, L. (2009). Aspectos psicosociales en universitarias embarazadas. Revista de Salud Pública, 11(6), 988-998. https:// doi.org/10.1590/S0124-00642009000600015

Estupiñán, M. R. y Vela C. D. R. (2012). Calidad de vida de madres adolescentes estudiantes universitarias. Revista Colombiana de Psiquiatría, 41(3), 536-549.

García Hernández, G. H. (2014). Embarazo adolescente y pobreza, una relación compleja. Iztapalapa. Revista de ciencias sociales y humanidades, 35(77), 13-53. https://doi.org/10.28928/ri/772014/atc1/ garciahernandeze

Hernández, A. y Rojas, H. (2018). El mercado del cuidado, el cuidado del mercado de la niñez en perspectiva de los cuidadores. Saber, Ciencia y Libertad, 13(2), 21-32. https://doi. org/10.18041/2382-3240/saber.2018v13n2.4633

Hernández, K. y Orozco, E. (2011). Embarazo en estudiantes de carreras universitarias. Dictamen Libre, 8(8), 68-73.

Huerta Mata, R. M. (2019). Las jóvenes madres solteras universitarias: Apoyo en el cuidado de los(as) hijos(as). Cultura de los Cuidados (Edición digital), 23 (54). http://dx.doi. org/10.14198/cuid.2019.54.19

Mazuera A., R. y Albornoz A., N. (2017). Maternidad adolescente, desigualdad social y exclusión educativa en el Norte de Santander (Colombia) y Táchira (Venezuela). Espacio Abierto, 26(1), 121-137.

Ministerio de Salud y Protección Social. (2014). Política Nacional de Sexualidad, Derechos Sexuales y Derechos Reproductivos. Bogotá: 
MSPS. Recuperado de: https://www.minsalud. gov.co/sites/rid/Lists/BibliotecaDigital/RIDE/ DE/LIBRO\%20POLITICA\%2OSEXUAL\%20SEPT\%20 10.pdf

Molinero, P. J., Morales, J., Vega, L., Montoro, J., Linares, M. y Álvarez, C. (2007). Actitud y adaptación maternal en el embarazo. Cultura de los cuidados, XI(21), 28-32. http://doi. org/10.14198/cuid.2007.21.05

Morales, D. E., Solanelles R. A. M., Mora G. S. R. y Miranda, G. O. (2013). Embarazo no deseado en alumnas universitarias. Revista Cubana de Medicina Militar, 42(2), 153-163.

Naciones Unidas. (1994). Informe de la Conferencia Internacional sobre la Población y el Desarrollo. El Cairo: UNFPA. Recuperado de: https:// www.unfpa.org/sites/default/files/pub-pdf/ icpd_spa.pdf

Núñez, D., Chavarría, V. y Molina, J. (2011). Influencia de los factores socioeconómicos en la deserción estudiantil de la carrera de ciencias sociales. Ciencia E Interculturalidad, 6(1), 72-84. https://doi.org/10.5377/rci.v6i1.282

Palomar, C. (2009). Maternidad y mundo académico. Alteridades, 19(38), 55-73.

Preciado, F., Acuña, M. y García, M. (2005). Repercusiones del embarazo y la maternidad en la vida académica de las estudiantes universitarias. Estadística escolar de la Facultad de Pedagogía. Universidad de Colima, 125-132.

Profamilia. (2013). Factores protectores y de riesgo del embarazo adolescente en Colombia. Bogotá: Profamilia.

Rodríguez, G., Gil, J. y García, E. (1996). Metodología de la investigación cualitativa. Granada: Ediciones Algibe.

Rodríguez Urrego, M. (2019). La investigación sobre deserción universitaria en Colombia 2006-2016. Tendencias y resultados. Pedagogía y Saberes, 51. https://doi.org/10.17227/pys.num51-8664

Strauss, A. y Corbin, J. (1998). Basics of qualitative research. Techniques and procedures for developing grounded theory. Londres: Sage Publications Inc.

Toneli, M. J. (2006). Paternidad y juventud: investigando el universo de estratos populares en $\mathrm{El}$ Sur de Brasil. La ventana. Revista de estudios de género, 3(23), 213-236.

Velásquez, M. (2009). El embarazo no planificado y sus efectos sobre el rendimiento académico en las estudiantes de la Escuela de Ciencias Sociales, Universidad de Oriente, Núcleo de Sucre, Semestre II 2007 y I 2008. (tesis de pregrado). Universidad de Oriente. 